\title{
Quantum Dissipative Rashba Spin Ratchets
}

\author{
Sergey Smirnov, ${ }^{1}$ Dario Bercioux,,${ }^{1,2}$ Milena Grifoni, ${ }^{1}$ and Klaus Richter ${ }^{1}$ \\ ${ }^{1}$ Institut für Theoretische Physik, Universität Regensburg, D-93040 Regensburg, Germany \\ ${ }^{2}$ Physikalisches Institut, Albert-Ludwigs-Universität, D-79104 Freiburg, Germany
}

(Received 20 March 2008; revised manuscript received 29 April 2008; published 11 June 2008)

\begin{abstract}
We predict the possibility to generate a finite stationary spin current by applying an unbiased ac driving to a quasi-one-dimensional asymmetric periodic structure with Rashba spin-orbit interaction and strong dissipation. We show that under a finite coupling strength between the orbital degrees of freedom the electron dynamics at low temperatures exhibits a pure spin ratchet behavior, i.e., a finite spin current and the absence of charge transport in spatially asymmetric structures. It is also found that the equilibrium spin currents are not destroyed by the presence of strong dissipation.
\end{abstract}

DOI: 10.1103/PhysRevLett.100.230601

An opportunity to induce a net stationary particle current by unbiased external forces applied to a quantum dissipative one-dimensional (1D) periodic structure is provided when the system does not possess a center of inversion in real space [1]. Then the particle transport occurs due to the ratchet effect and the device works as a Brownian motor [2]. In the deep quantum regime the charge ratchet effect can only be achieved when at least the two lowest Bloch bands contribute to transport [3].

Recently a new research field of condensed matter physics, spintronics, has emerged. One of its central issues is how to generate pure spin currents (SC) in paramagnetic systems due to only spin-orbit interactions and without applied magnetic fields. Rashba spin-orbit interaction (RSOI) [4] represents one of the possible tools to reach this goal since the spin-orbit coupling strength can be externally controlled by a gate voltage. One way to get pure SC is due to the intrinsic spin-Hall effect $[5,6]$ expected in a high-mobility two-dimensional semiconductor systems with RSOI [7]. Such pure SC were experimentally detected through the reciprocal spin-Hall effect in Ref. [8]. An alternative is to induce pure SC through absorption of polarized light [9]. The generation of pure SC by coherent spin rectifiers [10] has been discussed only recently for a finite size setup with RSOI. However, the presence of dissipation has not been considered up to now.

In this Letter we address the challenging task of how to implement devices which can work both as Brownian charge and spin motors. Here a natural and also principle question for spintronics arises: Is it possible to switch a device working as a charge ratchet to a pure spin ratchet mode where the charge current (CC) is completely blocked? As mentioned above, when in a dissipative system without RSOI transport is restricted to only one Bloch band, the charge ratchet mechanism does not exist [3]. Whether the same effect takes place in a dissipative system with RSOI is an open and nontrivial question. In fact, the Rashba Hamiltonian is not invariant under reflection of a transport direction. Thus the Rashba Hamiltonian itself already has a built-in spatial asymmetry which due to the spin-orbit coupling can be further mixed with the periodic
PACS numbers: 05.60.Gg, 03.65.Yz, 72.25.Dc, 73.23.-b

potential symmetry or asymmetry. The presence of dissipation additionally increases the complexity of the problem because the influence of a dissipative environment on the orbital motion changes through RSOI the spin dynamics.

In this work we focus on the moderate-to-strong dissipation case and address how to implement a device which under influence of unbiased external ac driving yields a finite stationary spin current and at the same time blocks the directed stationary charge transport. To concretize our idea of a Brownian spin motor we consider a dissipative periodic system with RSOI and show that the spin-orbit interaction alone is not enough to produce SC: The system must additionally lack the spatial symmetry and its orbital degrees of freedom must be coupled.

The full Hamiltonian of our problem is $\hat{H}_{\text {full }}(t)=\hat{H}+$ $\hat{H}_{\text {ext }}(t)+\hat{H}_{\text {bath }}$, where $\hat{H}$ is the Hamiltonian of the isolated periodic system, $\hat{H}_{\text {ext }}(t)$ describes an external driving, and $\hat{H}_{\text {bath }}$ is responsible for dissipative processes.

The isolated quasi-1D periodic system is formed in a two-dimensional electron gas (2DEG) with RSOI using a periodic potential along the $x$ axis and a harmonic confinement along the $z$ axis:

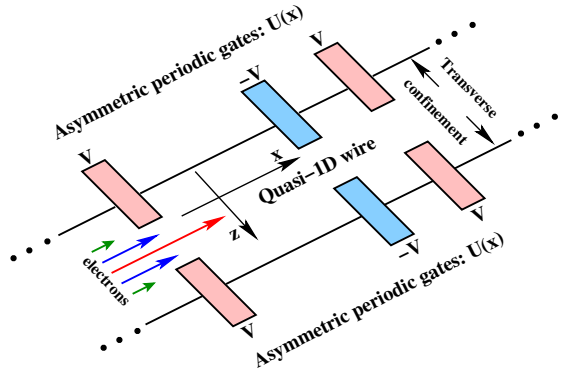

FIG. 1 (color online). A schematic picture of the isolated asymmetric periodic quasi-1D structure described by the Hamiltonian (1). In the center of the quasi-1D wire the periodic potential is weaker and gets stronger closer to the edges. Thus the electron group velocity is higher in the central region and tails off away from the center. 


$$
\hat{H}=\frac{\hbar^{2} \hat{\mathbf{k}}^{2}}{2 m}+\frac{m \omega_{0}^{2} \hat{z}^{2}}{2}-\frac{\hbar^{2} k_{\mathrm{so}}}{m}\left(\hat{\sigma}_{x} \hat{k}_{z}-\hat{\sigma}_{z} \hat{k}_{x}\right)+U_{\gamma}(\hat{x}, \hat{z}),
$$

where $U_{\gamma}(\hat{x}, \hat{z})=U(\hat{x})\left(1+\gamma \hat{z}^{2} / L^{2}\right), \hat{\mathbf{k}}$ is related to the momentum operator as $\hat{\mathbf{p}}=\hbar \hat{\mathbf{k}}, \omega_{0}$ is the harmonic confinement strength, $k_{\mathrm{so}}$ the spin-orbit coupling strength, $U(\hat{x})$ the periodic potential with the period $L$, and $\gamma \geqslant 0$ the orbit-orbit coupling strength. This isolated structure is sketched in Fig. 1 as it could be realized by appropriate gate evaporation techniques applied to 2DEGs formed in III-V compounds.

The periodic structure is subject to an external homogeneous time-dependent electric field, $\mathbf{E}(t) \equiv E(t) \hat{e}_{x}$. It can be experimentally implemented using, for example, linearly polarized light. This yields $\hat{H}_{\text {ext }}=e E(t) \hat{x}$, where $e$ is the elementary charge. We use the time dependence $e E(t) \equiv F \cos \left(\Omega\left(t-t_{0}\right)\right)$, which is unbiased.

The system is also coupled to a thermal bath. We assume the transverse confinement to be strong enough so that the probabilities of the direct bath-excited transitions between the transverse modes are negligibly small. Thus the environment couples to the electronic degrees of freedom only through $\hat{x}$. Furthermore, in the spirit of the Caldeira and Leggett model [11], we consider a harmonic bath with bilinear system-bath coupling.

The dynamical quantities of interest are the ratchet charge and spin currents $J_{C, S}(t)$ given as the statistical average of the longitudinal charge and spin current operators, $J_{C, S}(t) \equiv \operatorname{Tr}\left[\hat{J}_{C, S} \hat{\rho}(t)\right]$, where $\hat{\rho}(t)$ is the reduced statistical operator of the system, that is the full one with the bath degrees of freedom traced out. The $\mathrm{CC}$ operator is $\hat{J}_{C}(t)=-e d \hat{x} / d t$ and for the SC operator we use the definition suggested in Ref. [12], $\hat{J}_{S}(t)=d\left(\hat{\sigma}_{z} \hat{x}\right) / d t$.

It is convenient to calculate the traces using the basis which diagonalizes both $\hat{x}$ and $\hat{\sigma}_{z}$, because this requires to determine only the diagonal elements of the reduced density matrix. As shown in Ref. [13], for a periodic system with RSOI the energy spectrum can be derived from the corresponding truly $1 \mathrm{D}$ problem without RSOI. This leads to so-called Bloch sub-bands. The 2DEG is assumed to be sufficiently dilute to neglect the Pauli exclusion principle in the temperature range of our problem. The upper limit of this temperature range is considered to be low enough so that only the lowest Bloch sub-bands are populated. The basis which diagonalizes $\hat{x}$ and $\hat{\sigma}_{z}$ becomes in this case discrete. The total number of the Bloch sub-bands is equal to the product of the number, $N_{B}$, of the lowest Bloch bands from the corresponding truly $1 \mathrm{D}$ problem without RSOI, the number, $N_{t}$, of the lowest transverse modes and the number of spin states. In this work we shall use the model with $N_{B}=1, N_{t}=2$. The total number of the Bloch subbands in our problem is thus equal to four. Using $N_{B}=1$ we also assume that the external field is weak enough and does not excite electrons to higher Bloch bands. The representation in terms of the eigen-states of $\hat{x}$ for a model with discrete $x$-values is called discrete variable representation (DVR) [3,14]. Let us call $\sigma$-DVR the representation in which both the coordinate and spin operators are diagonal. Denoting the $\sigma$-DVR basis states as $\{|\alpha\rangle\}$ and eigenvalues of $\hat{x}$ and $\hat{\sigma}_{z}$ in a state $|\alpha\rangle$ by $x_{\alpha}$ and $\sigma_{\alpha}$, respectively, the CC and SC are rewritten as $J_{C}(t)=-e \sum_{\alpha} x_{\alpha} \dot{P}_{\alpha}(t)$ and $J_{S}(t)=\sum_{\alpha} \sigma_{\alpha} x_{\alpha} \dot{P}_{\alpha}(t)$, where $P_{\alpha}(t) \equiv\langle\alpha|\hat{\rho}(t)| \alpha\rangle$ is the population of the $\sigma$-DVR state $|\alpha\rangle$ at time $t$.

We are interested in the long time limit $\bar{J}_{C, S}^{\infty}$ of the currents $\bar{J}_{C, S}(t)$, averaged over the driving period $2 \pi / \Omega$.

The advantage of working in the $\sigma$-DVR basis is that real-time path integral techniques can be used to trace out exactly the bath degrees of freedom $[15,16]$. Moreover, at driving frequencies larger than the ones characterizing the internal dynamics of the quasi-1D system coupled to the bath, the averaged populations $\bar{P}_{\alpha}(t)$ can be found from the master equation,

$$
\dot{\bar{P}}_{\alpha}(t)=\sum_{\beta,(\beta \neq \alpha)} \bar{\Gamma}_{\alpha \beta} \bar{P}_{\beta}(t)-\sum_{\beta,(\beta \neq \alpha)} \bar{\Gamma}_{\beta \alpha} \bar{P}_{\alpha}(t),
$$

valid at long times. In Eq. (2) $\bar{\Gamma}_{\alpha \beta}$ is an averaged transition rate from the state $|\beta\rangle$ to the state $|\alpha\rangle$.

The first task is thus to identify the $\sigma$-DVR basis. The eigenstates $\left|l, k_{B}, j, \sigma\right\rangle$ of $\hat{\sigma}_{z}$ were found in [13] for the case $\gamma=0$. The results obtained in [13] are straightforwardly generalized to our model since for $N_{t}=2$ the operator $\hat{z}^{2}$ (and any even power of $\hat{z}$ ) is effectively diagonal. The quantum numbers $l, k_{B}, j, \sigma$ stand for the Bloch band index, quasimomentum, transverse mode index and $z$ projection of the spin, respectively. As mentioned above $l=$ $1, j=0,1$. One further finds

$$
\begin{aligned}
\left\langle l^{\prime}, k_{B}^{\prime}, j^{\prime}, \sigma^{\prime}|\hat{x}| l, k_{B}, j, \sigma\right\rangle= & \delta_{j^{\prime}, j} \delta_{\sigma^{\prime}, \sigma} \\
& \times{ }_{j}\left\langle l^{\prime}, k_{B}^{\prime}+\sigma k_{\mathrm{so}}|\hat{x}| l, k_{B}+\sigma k_{\mathrm{so}}\right\rangle_{j},
\end{aligned}
$$

where the index $j$ under the bra and ket symbols indicates that the corresponding electronic states are obtained using the periodic potential $U_{\gamma, j}(x) \equiv U(x) \times$ $\left[1+\gamma \hbar(j+1 / 2) / m \omega_{0} L^{2}\right]$. For a fixed value of $j$ the diagonal blocks in Eq. (3) are unitary equivalent and thus the eigenvalues of $\hat{x}$ do not depend on $\sigma$. The eigenvalues of the matrix ${ }_{j}\left\langle l^{\prime}, k_{B}^{\prime}|\hat{x}| l, k_{B}\right\rangle_{j}$ are analytically found and have the form $x_{\zeta, m, j}=m L+d_{\zeta, j}$, where $m=$ $0, \pm 1, \pm 2 \ldots, \zeta=1,2, \ldots, N_{B}$ and the eigenvalues $d_{\zeta, j}$ are distributed within one elementary cell. Thus one can label the eigenstates of $\hat{x}$ as $|\zeta, m, j, \sigma\rangle$. The corresponding eigenvalues are $x_{\zeta, m, j, \sigma}=x_{\zeta, m, j}$. We see that the $\sigma$-DVR basis states $|\alpha\rangle$ introduced above are just the $|\zeta, m, j, \sigma\rangle$ states, that is $\{|\alpha\rangle\} \equiv\{|\zeta, m, j, \sigma\rangle\}$.

To calculate $\mathrm{CC}$ and $\mathrm{SC}$ we use the tight-binding approximation assuming that the matrix elements $\left\langle\zeta^{\prime}, m^{\prime}, j^{\prime}, \sigma^{\prime}|\hat{H}| \zeta, m, j, \sigma\right\rangle$ with $\left|m^{\prime}-m\right|>1$ are negligibly small. Let us introduce the definitions for the states 
$|m, \xi\rangle \equiv|\zeta=1, m, \xi\rangle$ where $\{\xi\}=\{(j, \sigma)\}$ and $\xi=1 \Leftrightarrow$ $(0,1), \quad \xi=2 \Leftrightarrow(0,-1), \quad \xi=3 \Leftrightarrow(1,1), \quad \xi=4 \Leftrightarrow$ $(1,-1)$. Correspondingly, we introduce hopping matrix elements $\Delta_{\xi^{\prime}, \xi}^{m^{\prime}, m} \equiv\left\langle m^{\prime}, \xi^{\prime}|\hat{H}| m, \xi\right\rangle \quad\left(m^{\prime} \neq m\right.$ and/or $\xi^{\prime} \neq$ $\xi$ ) and on-site energies $\varepsilon_{\xi} \equiv\langle m, \xi|\hat{H}| m, \xi\rangle$.

Because of the harmonic confinement and RSOI the system is split into two channels: one with $\xi=1,4$ and another with $\xi=2,3$. The two channels are independent of each other, that is, transitions between them are forbidden. This picture is general and valid for an arbitrary number of the transverse modes. For clarity we below only consider the channel with $\xi=1,4$. Two independent channels were also found for a different type of confinement in Ref. [17].

Assuming that the hopping matrix elements are small enough we can use the second-order approximation [3] for the averaged transition rates in Eq. (2). We have

$$
\begin{aligned}
\bar{\Gamma}_{\xi^{\prime}, \xi}^{m^{\prime}, m}= & \frac{\left|\Delta_{\xi^{\prime}, \xi}^{m^{\prime}, m}\right|^{2}}{\hbar^{2}} \int_{-\infty}^{\infty} d \tau \exp \left[-\frac{\left(x_{m, \xi}-x_{m^{\prime}, \xi^{\prime}}\right)^{2}}{\hbar} Q[\tau, J(\omega)]\right. \\
& \left.+i \frac{\varepsilon_{\xi}-\varepsilon_{\xi^{\prime}}}{\hbar} \tau\right] J_{0}\left[\frac{2 F\left(x_{m, \xi}-x_{m^{\prime}, \xi^{\prime}}\right)}{\hbar \Omega} \sin \left(\frac{\Omega \tau}{2}\right)\right],
\end{aligned}
$$

where $x_{m, \xi} \equiv x_{\zeta=1, m, \xi}=m L+d_{\xi}$ with $d_{\xi} \equiv d_{1, j}$. In Eq. (4) $J_{0}(x)$ denotes the zero-order Bessel function and $Q[\tau, J(\omega)]$ is the twice integrated bath correlation function being a function of time $\tau$ and a functional of the bath spectral density $J(\omega)[3,16]$. The dependence of the transition rates on the orbit-orbit coupling $\gamma$ comes from two sources. The first one is the Bloch amplitudes and the second is the difference $\Delta d \equiv d_{1,0}-d_{1,1}$. In a tightbinding model the periodic potential is strong and thus $\Delta d$ can be made less than all the relevant length scales, $\Delta d / l_{r} \ll 1$, where $l_{r}=\min \left[L, \sqrt{\hbar / m \omega_{0}}, \hbar \Omega / F\right]$. Hence the main effect of the orbit-orbit coupling on $\bar{\Gamma}_{\xi^{\prime}, \xi}^{m^{\prime}, m}$ comes only through the Bloch amplitudes, and we neglect terms of order $\mathcal{O}\left(\Delta d / l_{r}\right)$.

We then arrive at the main results of our work, the absence of the charge transport, $\bar{J}_{C}^{\infty}=0$, and the expression for the nonequilibrium spin current (NESC), $\bar{J}_{n-e, S}^{\infty} \equiv$ $\bar{J}_{S}^{\infty}-\bar{J}_{e, S}^{\infty}$ :

$$
\begin{aligned}
\bar{J}_{n-e, S}^{\infty}= & -2 L\left(\frac{I_{14} I_{41}}{I_{14}+I_{41}}-\frac{I_{14}^{(0)} I_{41}^{(0)}}{I_{14}^{(0)}+I_{41}^{(0)}}\right) \frac{k_{\mathrm{so}}^{2} \hbar^{3} \omega_{0}}{m} \\
& \times \sum_{k_{B}, k_{B}^{\prime}} \sin \left[\left(k_{B}-k_{B}^{\prime}\right) L\right] \operatorname{Im}\left[\mathcal{F}_{k_{B}, k_{B}^{\prime}}\right],
\end{aligned}
$$

where $I_{\xi^{\prime}, \xi}, I_{\xi^{\prime}, \xi}^{(0)}$ are the integrals from (4) with and without driving, $F \neq 0$ and $F=0$, respectively, and

$$
\begin{aligned}
\mathcal{F}_{k_{B}, k_{B}^{\prime}} \equiv & u_{\gamma, 0 ; 1, k_{B}+k_{\mathrm{so}}}^{\mathrm{DVR}}\left(d_{1,0}\right) u_{\gamma, 1 ; 1, k_{B}^{\prime}-k_{\mathrm{so}}}^{\mathrm{DVR}}\left(d_{1,1}\right) \\
& \times\left[u_{\gamma, 1 ; 1, k_{B}-k_{\mathrm{so}}}^{\mathrm{DVR}}\left(d_{1,1}\right) u_{\gamma, 0 ; 1, k_{B}^{\prime}+k_{\mathrm{so}}}^{\mathrm{DVR}}\left(d_{1,0}\right)\right]^{*},
\end{aligned}
$$

where $u_{\gamma, j ; 1, k_{B}}^{\mathrm{DVR}}\left(d_{1, j}\right)$ is the DVR Bloch amplitude of the first band for electrons in the periodic potential $U_{\gamma, j}(x)$.

In Eq. (5) we have eliminated from $\bar{J}_{S}^{\infty}$ the equilibrium spin current (ESC), $\bar{J}_{e, S}^{\infty}$, following Ref. [18]. The fact that the ESC turns out to be finite shows that the definition of SC suggested in Ref. [12] does not automatically eliminate the presence of ESC. However, as pointed out in Ref. [12], this current really vanishes in insulators. This can be seen from Eq. (5). When the potential is strong, electrons are localized, the dependence of the function $\mathcal{F}_{k_{B}, k_{B}^{\prime}}$ on the quasimomentum disappears, and as a result both ESC and NESC are equal to zero. This reasonable result is ensured by the spin current definition taking proper care of the spin torque. It is interesting to note that ESCs are present even in a system with strong dissipation. As recently proposed in Ref. [19], ESCs can effectively be measured using a Rashba medium deposited on a flexible substrate playing a role of a mechanical cantilever.

We can determine the conditions under which the SC is finite. First of all from Eq. (5) it follows that the spin-orbit coupling must be finite, i.e., $k_{\text {so }} \neq 0$. Further, from Eq. (6) one observes, that when $\gamma=0$, the Bloch amplitudes do not depend on $j, u_{\gamma=0, j ; 1, k_{B}}^{\mathrm{DVR}}\left(d_{1, j}\right) \equiv u_{1, k_{B}}^{\mathrm{DVR}}\left(d_{1}\right)$, and since $\left[u_{1, k_{B}}^{\mathrm{DVR}}\left(d_{1}\right)\right]^{*}=u_{1,-k_{B}}^{\mathrm{DVR}}\left(d_{1}\right)$ (time-reversal symmetry), the function $\mathcal{F}_{k_{B}, k_{B}^{\prime}}$ becomes even with respect to its arguments. Then from Eq. (5) one gets zero SC. Thus the second condition is the presence of the orbit-orbit coupling. Finally, since for a symmetric periodic potential the Bloch amplitudes are real functions, we conclude that the function $\mathcal{F}_{k_{B}, k_{B}^{\prime}}$ is also real in this case, that is $\operatorname{Im}\left[\mathcal{F}_{k_{B}, k_{B}^{\prime}}\right]=0$. As a result the third condition is the presence of spatial asymmetry.

Below we present corresponding numerical results. All energies and frequencies are given in units of $\hbar \omega_{0}$ and $\omega_{0}$, respectively. The parameters are taken for an InGaAs/InP quantum wire: $\hbar \omega_{0}=0.9 \mathrm{meV} ; \quad \alpha \equiv \hbar^{2} k_{\mathrm{so}} / m=$ $9.94 \times 10^{-12} \mathrm{eV} \cdot \mathrm{m} ; \quad m=0.037 m_{0}$, respectively. For $k_{\mathrm{so}} L=\pi / 2$ one gets $L=0.32 \mu \mathrm{m}$.

The dependence of the NESC on the amplitude of the external driving is shown in Fig. 2 for the asymmetric periodic potential (see inset) $U(x)=$ $\sum_{n=0}^{2} V_{n} \cos \left(2 \pi n x / L-\phi_{n}\right)$ with $V_{0}=4, \quad V_{1}=-V_{0}$, $V_{2}=3.89, \phi_{0}=\phi_{2}=0.0, \phi_{1}=1.9$. The gap between the Bloch bands with $l=1$ and $l=2$ is $\Delta E_{12} \approx 10.5$. In Fig. $2 F L, \hbar \Omega<\Delta E_{12}$ that is the numerical results are consistent with the theoretical model assumptions. As an example we have used an Ohmic bath with the spectral density $J(\omega)=\eta \omega \exp \left(-\omega / \omega_{c}\right)$, where the viscosity coefficient (in units of $m \omega_{0}$ ) is $\eta=0.25,0.5,0.75$ and the cutoff frequency is $\omega_{c}=10$. As it can be seen, the NESC has an oscillating nature. However, the oscillation amplitude goes down when the driving increases. Physically such behavior can be attributed to an effective renormalization of the band structure in a high-frequency electric field [15]. The group velocity decreases in a nonmonotonic 


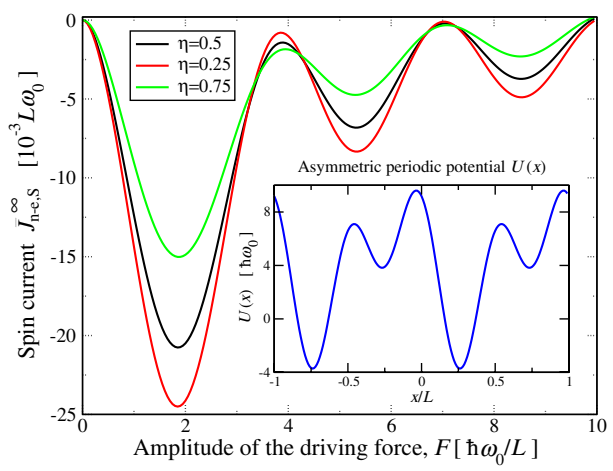

FIG. 2 (color online). Nonequilibrium spin current, $\bar{J}_{n-e, S}^{\infty}$, as a function of the amplitude, $F$, of the driving force for different values of the viscosity coefficient $\eta$. Temperature $k_{\text {Boltz. }} T=0.5$, spin-orbit coupling strength $k_{\mathrm{so}} L=\pi / 2$, orbit-orbit coupling strength $\gamma=0.1$, driving frequency $\Omega=1$. The inset displays the shape of the periodic potential.

way which, due to RSOI, slows down the spin kinetics. For increasing values of $\eta$ the dissipation induced decoherence in the system gets more pronounced. The system becomes more classical and thus the tunneling processes become less intensive. This leads to the spin current reduction which one observes in Fig. 2.

In Fig. 3 the NESC is plotted versus $k_{\mathrm{so}} L$ while $\gamma$ plays the role of a parameter. The oscillations of the NESC have minima located at $n G / 2$ where $n=0,1,2, \ldots$, and $G$ is the reciprocal lattice vector. Physically this reflects the fact that for those values of $k_{\mathrm{so}}$ the Rashba split becomes minimal due to the periodicity of the energy spectrum in the $\mathbf{k}$-space. The magnitude of these oscillations decreases with decreasing orbit-orbit coupling, and the current vanishes for $\gamma=0$.

In summary, we have studied stationary quantum transport in a driven dissipative periodic quasi-one-dimensional system with Rashba spin-orbit interaction and orbit-orbit

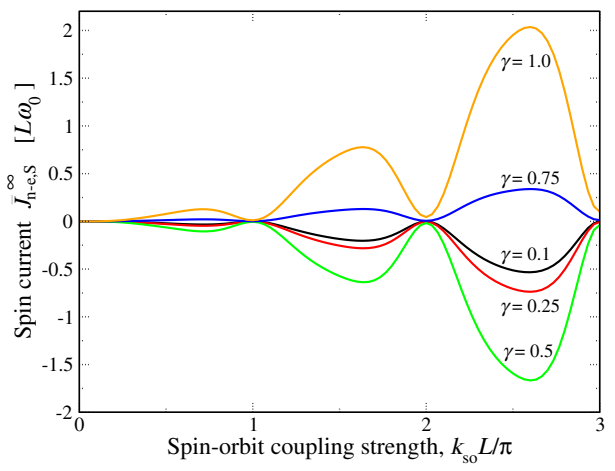

FIG. 3 (color online). Nonequilibrium spin current, $\bar{J}_{n-e, S}^{\infty}$, as a function of the spin-orbit coupling strength, $k_{\text {so }}$, for different values of the orbit-orbit coupling strength, $\gamma$. The driving amplitude and viscosity coefficient are $F=2 \hbar \omega_{0} / L, \eta=0.5$. The other parameters are as in Fig. 2. coupling. The spin ratchet effect has been investigated and an analytical expression for the spin current has been derived and analyzed. This analysis has revealed that for the case of moderate-to-strong dissipation the necessary conditions for nonvanishing spin currents are the spatial asymmetry of the periodic potential as well as a finite strength of the spin-orbit interaction and orbit-orbit coupling. It has been demonstrated that in a dissipative system equilibrium spin currents can exist. Our numerical calculations have shown characteristic oscillations of the spin current as a function of the amplitude of the driving force and the spin-orbit coupling strength. Finally, we note, that since the spin current has the in-plane polarization, it can be efficiently measured by a magneto-optic Kerr microscope using the cleaved edge technology as suggested recently in Ref. [20].

We thank J. Peguiron for useful discussions. Support from the DFG under the program No. SFB 689 is acknowledged.

[1] P. Reimann, M. Grifoni, and P. Hänggi, Phys. Rev. Lett. 79, 10 (1997).

[2] R. D. Astumian and P. Hänggi, Phys. Today 55, 33 (2002).

[3] M. Grifoni, M. S. Ferreira, J. Peguiron, and J. B. Majer, Phys. Rev. Lett. 89, 146801 (2002).

[4] E. Rashba, Fiz. Tverd. Tela (Leningrad) 2, 1224 (1960).

[5] S. Murakami, N. Nagaosa, and S.-C. Zhang, Science 301, 1348 (2003).

[6] J. Sinova, D. Culcer, Q. Niu, N. A. Sinitsyn, T. Jungwirth, and A. H. MacDonald, Phys. Rev. Lett. 92, 126603 (2004).

[7] For an experimental indication, see J. Wunderlich, B. Kaestner, J. Sinova, and T. Jungwirth, Phys. Rev. Lett. 94, 047204 (2005).

[8] S. O. Valenzuela and M. Tinkham, Nature (London) 442, 176 (2006).

[9] B. Zhou and S.-Q. Shen, Phys. Rev. B 75, 045339 (2007).

[10] M. Scheid, A. Pfund, D. Bercioux, and K. Richter, Phys. Rev. B 76, 195303 (2007).

[11] A. O. Caldeira and A. J. Leggett, Phys. Rev. Lett. 46, 211 (1981).

[12] J. Shi, P. Zhang, D. Xiao, and Q. Niu, Phys. Rev. Lett. 96, 076604 (2006).

[13] S. Smirnov, D. Bercioux, and M. Grifoni, Europhys. Lett. 80, 27003 (2007).

[14] D. O. Harris, G. G. Engerholm, and W. D. Gwinn, J. Chem. Phys. 43, 1515 (1965).

[15] M. Grifoni and P. Hänggi, Phys. Rep. 304, 229 (1998).

[16] U. Weiss, Quantum Dissipative Systems (World Scientific, Singapore, 1999), 2nd ed.

[17] C. A. Perroni, D. Bercioux, V.M. Ramaglia, and V. Cataudella, J. Phys. Condens. Matter 19, 186227 (2007).

[18] E. I. Rashba, Phys. Rev. B 68, 241315(R) (2003).

[19] E. B. Sonin, Phys. Rev. Lett. 99, 266602 (2007).

[20] P. Kotissek, M. Bailleul, M. Sperl, A. Spitzer, D. Schuh, W. Wegscheider, C. H. Back, and G. Bayreuther, Nature Phys. 3, 872 (2007). 